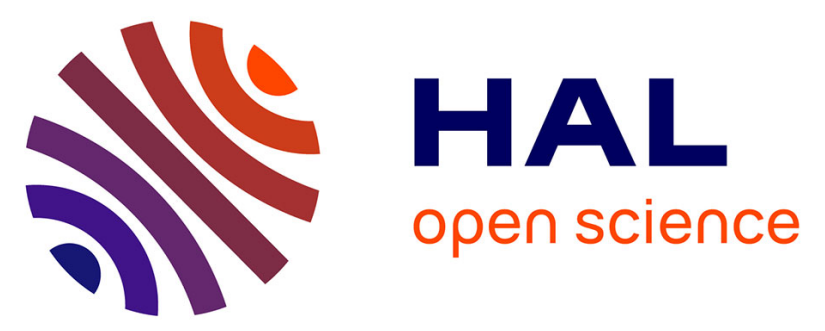

\title{
Large-eddy simulation of turbulent duct flow: heating and curvature effects
}

Jérôme Hebrard, Olivier Métais, Martin Salinas-Vazquez

\section{To cite this version:}

Jérôme Hebrard, Olivier Métais, Martin Salinas-Vazquez. Large-eddy simulation of turbulent duct flow: heating and curvature effects. International Journal of Heat and Fluid Flow, 2004, 25, pp.569580. 10.1016/S0142-727X(04)00046-3 . hal-00265144

\section{HAL Id: hal-00265144 \\ https://hal.science/hal-00265144}

Submitted on 19 Mar 2020

HAL is a multi-disciplinary open access archive for the deposit and dissemination of scientific research documents, whether they are published or not. The documents may come from teaching and research institutions in France or abroad, or from public or private research centers.
L'archive ouverte pluridisciplinaire HAL, est destinée au dépôt et à la diffusion de documents scientifiques de niveau recherche, publiés ou non, émanant des établissements d'enseignement et de recherche français ou étrangers, des laboratoires publics ou privés.

\section{(c)(1)}

Distributed under a Creative Commons Attribution| 4.0 International License 


\title{
Large-eddy simulation of turbulent duct flow: heating and curvature effects
}

\author{
Jérôme Hébrard ${ }^{\mathrm{a}, *}$, Olivier Métais ${ }^{\mathrm{a}}$, Martin Salinas-Vasquez ${ }^{\mathrm{b}}$ \\ ${ }^{a}$ L.E.G.I./Institut de Mécanique de Grenoble, B.P. 53, 38041 Grenoble Cedex 09, France \\ ${ }^{\mathrm{b}}$ Instituto de Ingenieria, Unam, Mexico
}

Large eddy simulations of the turbulent gas flow within ducts of square cross-section are performed. The spatial development of turbulent flow inside a heated straight duct is first considered with a higher temperature suddenly imposed at one of the duct walls. The downstream development of the thermal boundary layer is then studied and compared with the fully developed turbulent case. The gradual increase with temperature of the viscosity near the heated wall yields a progressive enhancement of the turbulent structures and to a single ejection localized near the middle plane of the heated wall.

The use of curvilinear coordinates allows to consider ducts of more complex geometries and to investigate the combined effects of heating and curvature. The case of an S-shape duct is then considered exhibiting both convex and concave curvatures. The pressure gradient between the inner and outer wall of the curved sections leads to the apparition of intense counter-rotating Dean vortices associated with an intense transverse flow with a maximum intensity of $22 \%$ of the bulk velocity. Downstream of the second curved section of the duct, the flow exhibits a complex distribution of various vortices. In the heated case, the mutual interaction between the heating and the Dean vortices is investigated. The heating is seen to enhance both the size and intensity of the Dean vortices when situated close to the heated wall.

Keywords: Turbulence; Large eddy simulation; Heat transfer; Curvature

\section{Introduction}

For numerous engineering applications, it is important to reach a deeper understanding and a better prediction of the heat exchanges between a heated wall and the adjacent turbulent flow. Among these applications we are particularly interested in the enhancement of the heat exchanges taking place within the ducts present in the wall of the rocket engines to cool the system. For these applications, it is important to note that the intensity of the heat fluxes is very high. As far as numerical modelling is concerned, the statistical mod-

\footnotetext{
${ }^{*}$ Corresponding author.

E-mail addresses: hebrard@hmg.inpg.fr (J. Hébrard), metais@hmg.inpg.fr, olivier.metais@hmg.inpg.fr (O. Métais),msv@quetzal.iingen.unam.mx (M. Salinas-Vasquez).
}

elling approach heavily relies on empirical laws to mimic the near wall flow behaviour and the near wall heat fluxes. In previous works, we have successfully applied the large-eddy simulation (LES) technique based on the structure function subgrid-scale model (see Lesieur and Métais, 1996) to study both the statistical characteristics and the instantaneous three-dimensional structures of the turbulent flow through a straight heated duct (see Salinas-Vasquez and Métais, 2001, 2002) at Mach $=0.5$. In the work by Salinas-Vasquez and Métais (2002) (referred to as SM), the temperature was imposed at all the walls and a higher temperature value was prescribed on one of the walls (referred to as the heated wall). These boundary conditions for the temperature are compatible with the use of periodic boundary conditions in the streamwise direction. This periodic approach presents a double advantage: first, it allows for a reasonably rapid convergence of the statistical quantities, since the streamwise flow homogeneity can be used. Second, for subsonic flows, periodic boundary conditions are known 
to be well-posed from a numerical view point, at variance with open boundary conditions. However, in several industrial devices involving heated boundary layers, a given heat-flux is actually imposed at the heated wall and the turbulent flow can no longer be assumed periodic in the streamwise direction due to the continuous energy increase along this direction. One has then to deal with spatially developing turbulent flows which require a proper prescription of inflow and outflow boundary conditions: this is a challenging problem for unsteady subsonic flows which has been previously investigated by several authors (see e.g. Poinsot and Lele, 1992; Grinstein, 1994).

Except for the work by SM, there exist no direct numerical simulations and no LES devoted to the study of the heat exchanges within closed heated ducts. In a first part, we extend SM's work by simulating the spatial growth of the thermal boundary layer over a heated wall. As pointed above, this configuration is in closer correspondence with real industrial situations than a fully developed thermal state.

The second part is devoted to the study of the combined effects of curvature and heating. Curvature effects are indeed often present in heat exchangers: this is the case for the cooling ducts in the walls of the combustion chamber and of the divergent section of a rocket engine. We here consider an S-shape duct which mimics the double curvature effect encountered in the cooling ducts of a combustion chamber. Although this constitutes a simplified geometry, this study allows one to reach a better understanding of the turbulent structures responsible for the heat exchanges and will serve as a database to test and hopefully improve the empirical laws used in classical statistical models of turbulence. To our knowledge, no experimental data are available concerning the heated curved duct: this unfortunately makes any experimental validation impossible. Note that the duct flow is a very challenging problem for the classical statistical models of turbulence since it is characterized by weak secondary flows which cannot be reproduced by classical eddy-viscosity models of $k-\epsilon$ type. A correct prediction of the curvature effects and of the flow modifications associated with heating represents an even bigger challenge for statistical modelling.

\section{Numerical configuration}

All the ducts simulated here have a square crosssection and $D_{\mathrm{h}}$ designates the hydraulic diameter. We use the same numerical code as the one used by SalinasVasquez and Métais (2002) which solves the threedimensional compressible Navier-Stokes equations. The system of equations is solved in generalized curvilinear coordinates (see Salinas-Vasquez and Métais, 2002 for details). We use the same corrector-predictor scheme as the one proposed by Gotlieb and Turkel (1976), but the finite difference approximation is replaced here by the compact scheme under McCormack's formulation, second order in time and fourth order in space, devised by Kennedy and Carpenter (1997). All simulations are performed at a bulk Reynolds $R e_{\mathrm{b}}=6000$ and Mach $=$ 0.5 . The Reynolds and Mach numbers are based on the bulk velocity $\left(U_{\mathrm{b}}\right)$, the bulk density $\left(\rho_{\mathrm{b}}\right)$, the hydraulic diameter $\left(D_{\mathrm{h}}\right)$ and the wall temperature $\left(T_{\mathrm{w}}\right)$.

As previously pointed out, it is a challenging problem to impose proper boundary conditions for subsonic unsteady flows which exhibit a spatial growth. First, to obtain a fully developed turbulent field at the entry of our computational domain, the inlet conditions for the spatially growing duct flow are provided, at each time step, by a periodic isothermal duct which is concurrently computed (see Fig. 1).

The periodic duct boundary conditions at the walls are no-slip and isothermal with an imposed temperature $T_{\mathrm{w}}$. The wall pressure is obtained by solving the NavierStokes equations at the wall. For the spatial duct, the wall boundary conditions are also no-slip and the wall pressure is determined as previously. The spatial duct is linked to the periodic duct by the characteristic boundary conditions proposed by Poinsot and Lele (1992) (see also Poinsot and Veynante, 2001). The velocity and the temperature field computed at the last section of the periodic duct is used to specify the inflow conditions at the first section of the spatial duct. At the outlet, the subsonic partially reflective outflow boundary conditions have been implemented (see Poinsot and Veynante, 2001).

For the straight duct, the size of the computational domain is taken equal to $31 D_{\mathrm{h}} \times D_{\mathrm{h}} \times D_{\mathrm{h}}$ for the spatial duct and $6.4 D_{\mathrm{h}} \times D_{\mathrm{h}} \times D_{\mathrm{h}}$ for the precursory periodic duct with the first length corresponding to the streamwise direction and the other two to the two transverse directions. The corresponding number of grid points are respectively $318 \times 50 \times 50$ and $64 \times 50 \times 50$. Through grid refinement, we have checked that the results are almost independent of the grid resolution.

In the case of the S-shape duct, the size of the domain is taken equal to $15 D_{\mathrm{h}} \times D_{\mathrm{h}} \times D_{\mathrm{h}}$. The corresponding number of grid points are respectively $160 \times 50 \times 50$. The precursory periodic duct is identical to the previous case.

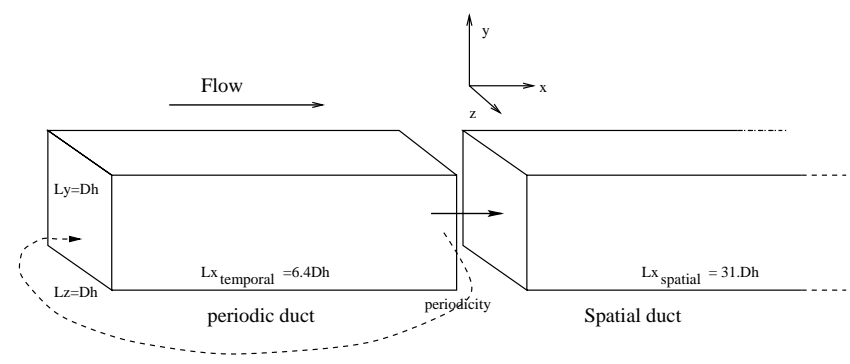

Fig. 1. Numerical configuration. 


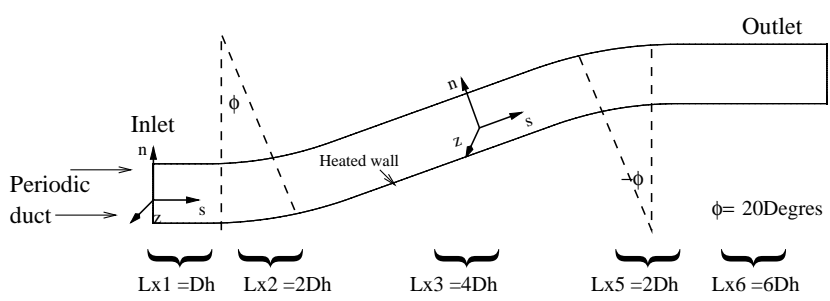

Fig. 2. S-shape duct configuration.

Note that the S-shape duct possesses two curved wall: one with a succession of a concave curvature followed by a convex curvature when the opposite wall exhibits a convex-concave curvature. We here use a right-handed curvilinear coordinates system $s, n$ and $z$ where $s$ follows the flow direction; $n$ corresponds with the direction normal to the curved walls with $n / D_{\mathrm{h}}=0$ taken on the concave-convex wall; $z$ is normal to the lateral walls. The Dean number constitutes the characteristic number for closed flows submitted to curvature effects: in the present case, $D_{n}=\operatorname{Re} \times\left(D_{\mathrm{h}} / R_{\mathrm{c}}\right)^{1 / 2} \approx 2353$ where $R_{\mathrm{c}}=6.5$ is the curvature radius on the duct centerline. A hyperbolic stretching is used in the $n$ and $z$ directions and the distance of the first grid point from the walls is given by $y / D_{\mathrm{h}}=z / D_{\mathrm{h}}=0.0046$ (straight duct) and $n / D_{\mathrm{h}}=z / D_{\mathrm{h}}=0.0046 \quad$ (S-shape duct) corresponding with $y^{+}=z^{+}=n^{+}=1.8$ in wall units of the precursory periodic duct. The detailed configuration for the S-shape duct is described in Fig. 2.

As far as the mean quantities are concerned, $\langle f\rangle(x, y, z)$ (resp. $\langle f\rangle(s, n, z)$ ) stands for the time average of the variable $f$. The bulk quantity $f_{\mathrm{b}}$ refers to the mean quantity $\langle f\rangle$ integrated along both transverses directions at a given cross-section $x$ (straight duct) or $s$ (S-shape duct): $f_{\mathrm{b}}$ is therefore a function of the streamwise location. We note the root mean square quantities as $u_{\mathrm{rms}}, v_{\mathrm{rms}}, w_{\mathrm{rms}}, T_{\mathrm{rms}}$ and the Reynolds stress components as $\left\langle u^{\prime} v^{\prime}\right\rangle,\left\langle u^{\prime} w^{\prime}\right\rangle$ and $\left\langle v^{\prime} w^{\prime}\right\rangle$.

\section{Results}

\subsection{Straight duct}

As a validation of our numerical procedure, a spatial duct with all its walls at the imposed temperature $T_{\mathrm{w}}$ has first been considered. A correct prescription of the outlet boundary conditions should yield a flow statistically identical with the flow in an isothermal periodic duct as the one studied through direct numerical simulation (DNS) by Gavrilakis (1992) and the statistics should therefore be independent of the streamwise direction. We have checked that, despite the relatively low resolution of our LES, a very good concordance is observed between our computation and the DNS results of
Gavrilakis (1992) both for the first and second order statistics.

We next study the spatial development of the thermal boundary. The temperature of one of the four walls is abruptly changed from $T_{\mathrm{w}}$ to $T_{\mathrm{h}}=2.5 T_{\mathrm{w}}$ for $x / D_{\mathrm{h}} \geqslant 0.4$. The other three walls have a constant temperature $T_{\mathrm{w}}$ over the whole length of the duct. As shown by SM, an important factor in the flow development is the viscosity enhancement due to the temperature increase close to the heated wall which induces a global augmentation of the characteristic size of the turbulent boundary layer structures. For high enough heating, SM showed that, in the periodic duct, the size of the flow ejections from the heated wall becomes such that these concentrate near the duct middle plane and tend to disappear in the other regions. The advantage of a spatially growing

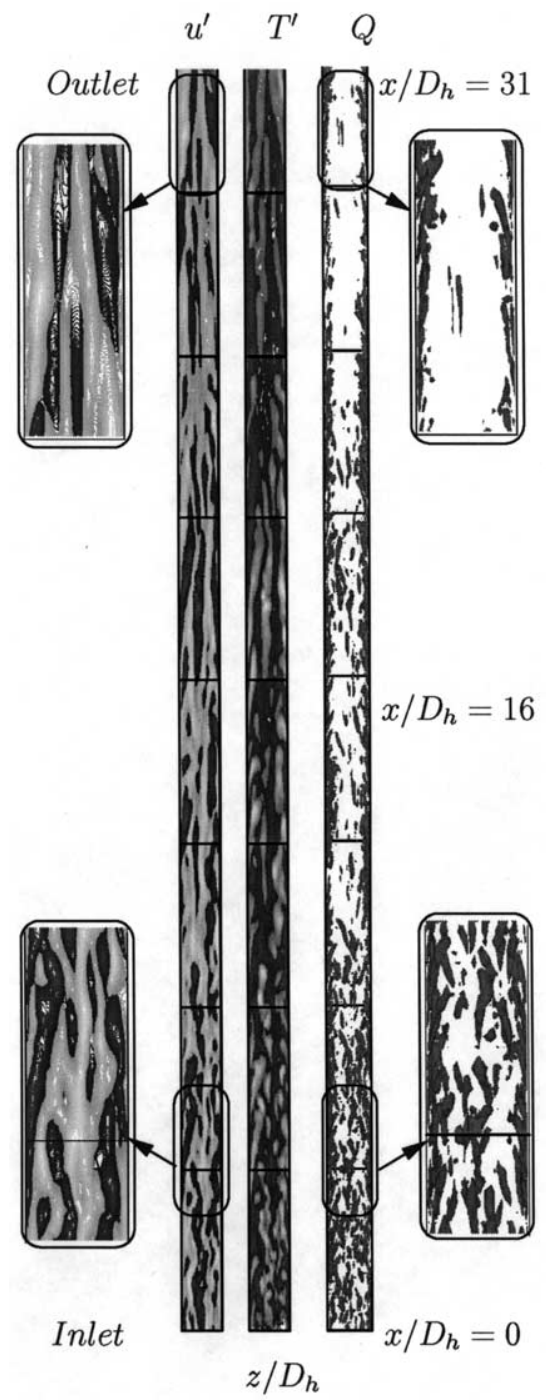

Fig. 3. Turbulent structures in the straight heated duct. Left: Fluctuant streamwise velocity near the hot wall, zooms near the inlet and the outlet of the duct. Middle: Fluctuant temperature near the hot wall. Right: Instantaneous $Q$ isosurfaces $Q=0.5\left(U_{\mathrm{b}} / D_{\mathrm{h}}\right)^{2}$ on the hot wall, zooms near the inlet and the outlet of the duct. 
simulation is the ability to visualize the progressive change of the flow structures near the heated wall. Fig. 3 shows a top view looking at the heated wall of the fluctuating fields for the temperature and the streamwise velocity in a plane parallel to the heated wall in the vicinity of the latter at $y / D_{\mathrm{h}}=0.01\left(y^{+}=4\right)$. Positive streamwise fluctuations (in light grey) correspond to fast fluid transported towards the wall (sweeps) and negative streamwise fluctuations (in dark grey) are associated with slow fluid ejected from the wall (ejections). The former correspond to cold fluid transported towards the hot wall and are consequently associated with negative temperature fluctuations (in dark grey) and the latter correspond to hot fluid ejected into the colder outer region (positive temperature fluctuations in light grey). Near the duct inlet, we recognize the characteristic system of low and high speed streaks with their elongated and narrow shape. From $x / D_{\mathrm{h}} \approx 8.0$, these become longer and wider as we move downstream and eventually their width is such that only two or three streaks are visible near the duct outlet. It is now well recognized that the $Q$ criterion based upon the second invariant of the velocity derivative tensor is a good way to identify the coherent structures. We recall that $Q=$ $\frac{1}{2}\left(\Omega_{i j} \Omega_{i j}-S_{i j} S_{i j}\right)$ with $\Omega_{i j} \Omega_{i j}$ the antisymmetric and $S_{i j} S_{i j}$ the symmetric part of the velocity gradient tensor (Hunt et al., 1988; Dubief and Delcayre, 2000). Fig. 3 shows that the turbulent structures, identified with the positive $Q$ isosurfaces, are very numerous at the inlet, but their number decreases in function of the streamwise direction. They concentrate around the middle wall plane and their longitudinal length is larger near to the outlet. It is well known that the near wall structures such as the streaks and the ejections scale with the viscous length. The progressive enhancement of the size of the turbulent structures is therefore directly linked with the progressive viscosity increase due to the temperature augmentation as we move downstream. By the end of the duct, only one ejection from the heated wall can occur and it is situated in the vicinity of the duct middle plane. The progressive formation of this central ejection is clearly illustrated in Fig. 4 which displays the
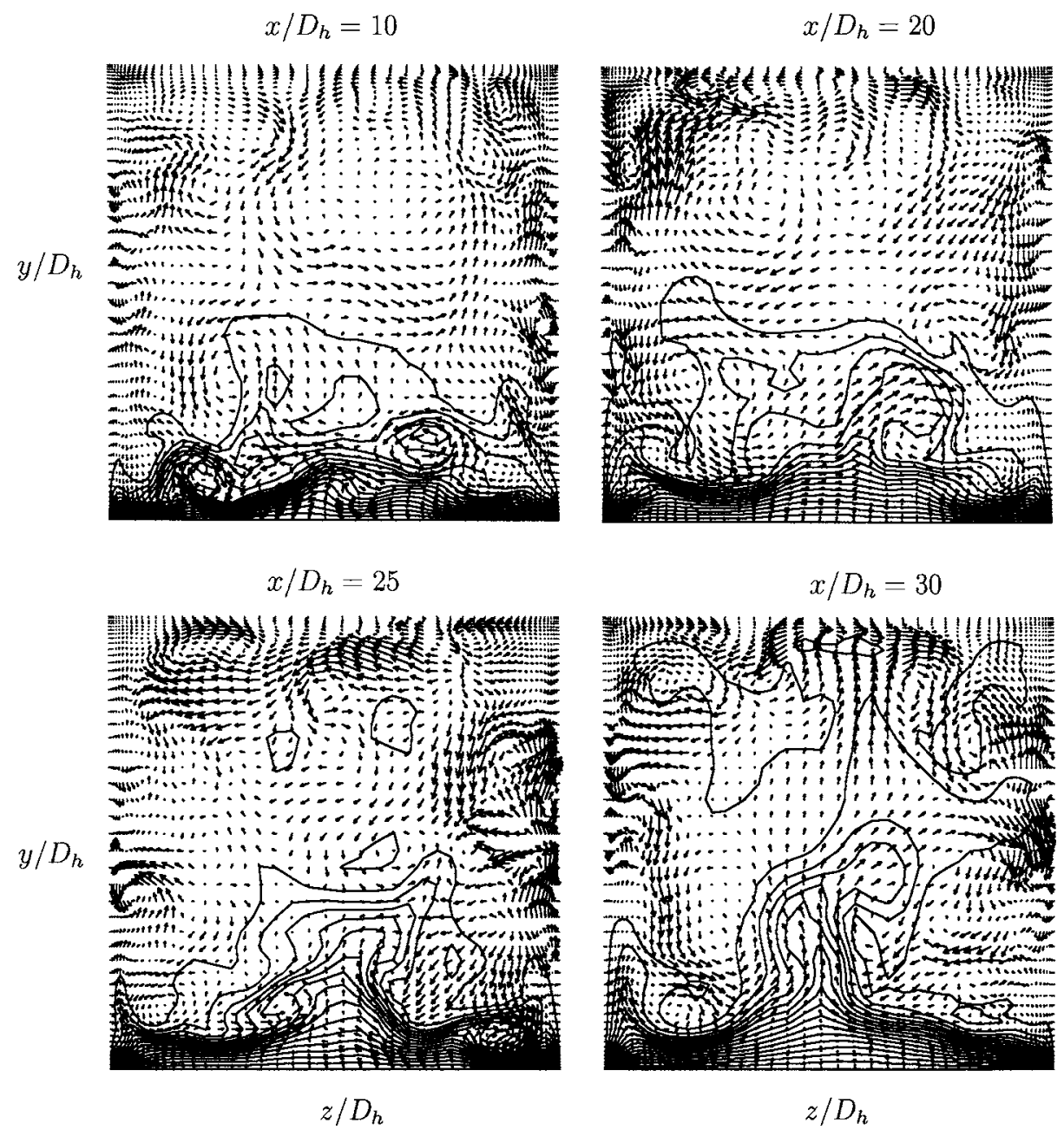

Fig. 4. Instantaneous temperature and transversal velocity vector fields for $x / D_{\mathrm{h}}=10,20,25$ and 30 . 
instantaneous temperature contours and the instantaneous transversal velocity vectors at four different $x$ cross-sections. For increasing downstream distance, we assist in the expulsion of hot fluid towards the duct core from the near wall region situated near the middle plane of the heated wall. This ejection is associated with a secondary flow transverse to the duct longitudinal direction consisting of fluid going away from the heated wall near the duct central plane bordered with cold fluid going towards the hot wall in a mushroom shape fashion. The downstream formation of this secondary flow is illustrated by Fig. 5 which shows the mean velocity field projected on several cross-sections for increasing $x$. From the left to the right of Fig. 5, $x / D_{\mathrm{h}}=0,10,20$ and
30. Note that $x / D_{\mathrm{h}}=0$ correspond to the non-heated case.

The secondary flow is progressively amplified both in intensity and size and reaches an intensity of $2.2 \%$ of $U_{\mathrm{b}}$ near the duct inlet up to $3.5 \%$ near the duct outlet. The gradual downstream development of the ejections is confirmed by Fig. 6 which displays the $-\left\langle u^{\prime} v^{\prime}\right\rangle$ profiles as a function of $y / D_{\mathrm{h}}$ at various $x$-locations. This Reynolds stress component is a well known tracer of the ejections and sweeps mechanisms and SM demonstrated the strong enhancement of this component in the periodic heated duct. The viscosity increase associated with the heating reduces the turbulence in the near wall region. Consequently, the amplitude of $-\left\langle u^{\prime} v^{\prime}\right\rangle$ is progressively

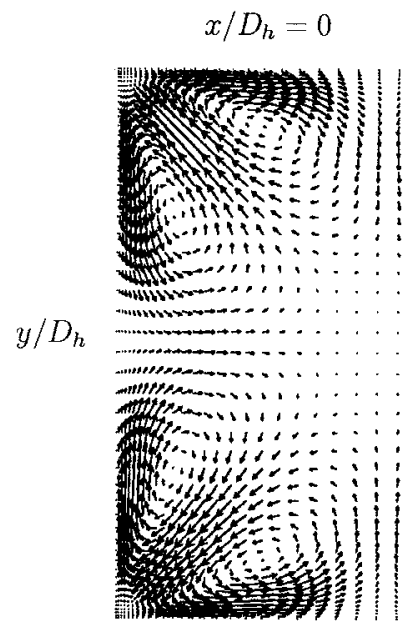

$x / D_{h}=10$

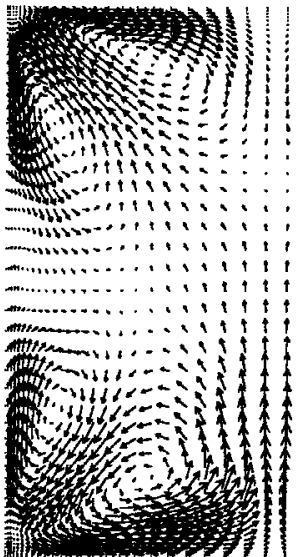

$x / D_{h}=20$

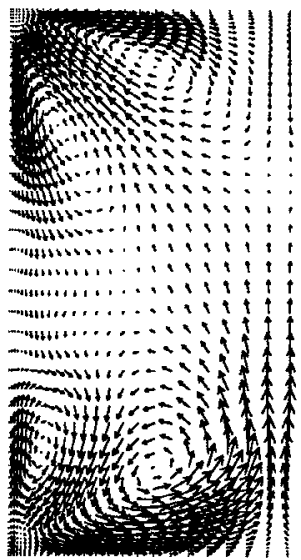

$z / D_{h}$

$$
x / D_{h}=30
$$

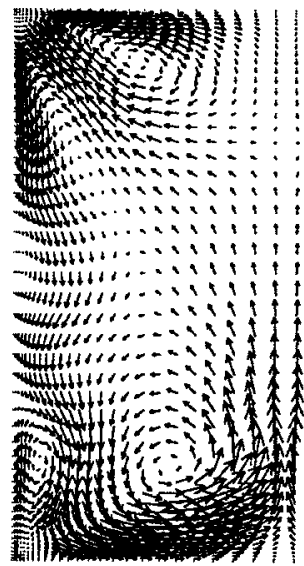

Fig. 5. Mean transversal velocity vector fields in a half section for $z / D_{\mathrm{h}}$ extending from 0 to 0.5 at $x / D_{\mathrm{h}}=0,10,20$ and 30 (from the left to the right).
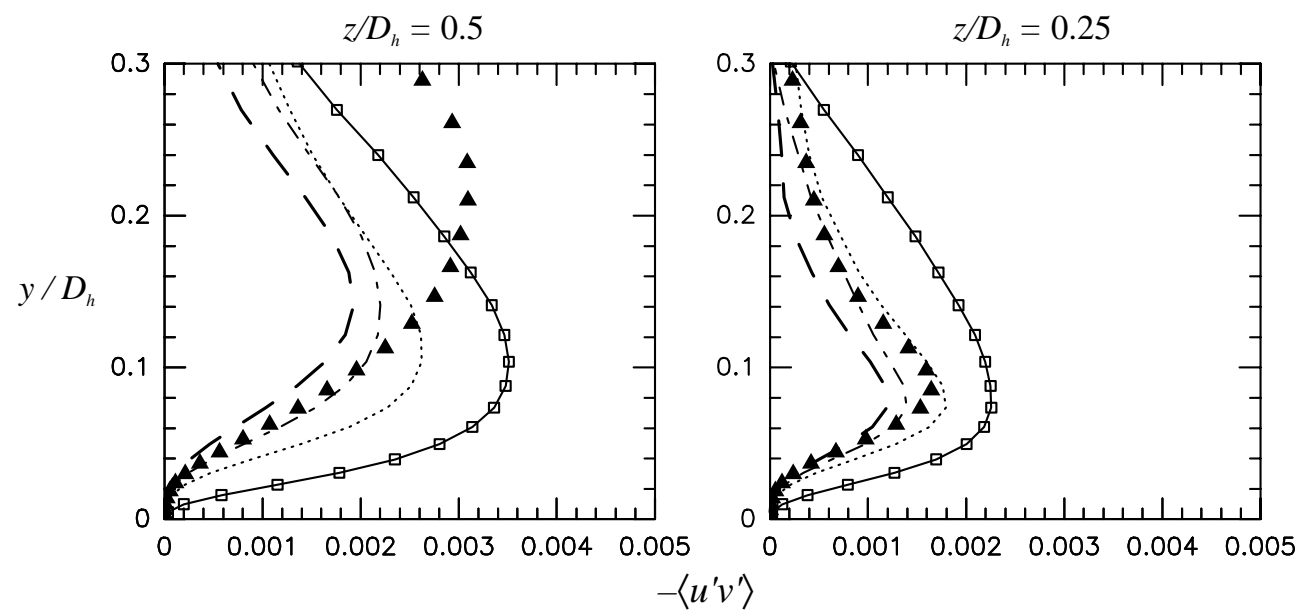

Fig. 6. Zoom on $-\left\langle u^{\prime} v^{\prime}\right\rangle$ profiles close the hot wall as a function of the distance $y / D_{\mathrm{h}}$ from the wall and at two fixed distances $z / D_{\mathrm{h}}=0.5$ and $z / D_{\mathrm{h}}=0.25$ from the lateral wall. Periodic heated duct $(\boldsymbol{\Delta})$; periodic non-heated duct $(\square)$. Spatial duct for different distances from the inlet: $x / D_{\mathrm{h}}=0$ $(-) ; x / D_{\mathrm{h}}=15(\cdots) ; x / D_{\mathrm{h}}=23(--) ; x / D_{\mathrm{h}}=30(--)$. Values are normalized by the local bulk velocity. 
reduced as we move downstream of the spatial duct. This is observed in both planes $z / D_{\mathrm{h}}=0.25$ and $z / D_{\mathrm{h}}=0.5$. In the duct middle plane $z / D_{\mathrm{h}}=0.5$, the maximum of the $-\left\langle u^{\prime} v^{\prime}\right\rangle$ profile is however progressively shifted away from the heated wall as the downstream distance increases: this indicates a gradual increase in the size of the ejections. Note that, even by the end of the duct, the size of the ejections is far from the one observed in the periodic heated duct of SM. Indeed, both the position and the amplitude of the $-\left\langle u^{\prime} v^{\prime}\right\rangle$ peak are lower than the values observed in the periodic case. This indicates that a fully developed thermal state takes a very long downstream distance to establish and that $31 D_{\mathrm{h}}$ are not sufficient to reach this state. In the plane $z / D_{\mathrm{h}}=0.25$, we assist in the progressive damping of $-\left\langle u^{\prime} v^{\prime}\right\rangle$ maximum and a decrease in the size of the ejections: this indicates than these are more and more concentrated around the duct central plane.

\subsection{S-shape duct}

Many works have been devoted to the study of the laminar flow within curved ducts focusing on the development of the coherent vortices called Dean vortices and the dependence of the flow structure on the Dean number (see e.g. Mees et al., 1996). As far as turbulent curved ducts are concerned, previous works were mainly devoted to comparisons between experimental works and statistical numerical modelling at high Reynolds numbers (see e.g. Sotiropoulos and Ventikos, 1998). Experimental works on the S-shape duct were performed by Bandyopadyhay and Ahmed (1993) or Bruns et al. (1999) in order to study the effect of two successive inverse curvatures. Since the Reynolds number in this experimental work is too high to be reached with our LES technique, only qualitative comparisons can be made: we have checked that our results provide the right trends as compared to experimental results.

We here consider a duct of square cross-section and concentrate on the turbulent structures present within the flow and the effect of heating on these structures. The previous works have shown that, both in the laminar and turbulent cases, the pressure gradient between the inner and outer wall of the curved section leads to the formation of an intense secondary flow constituted of large counter-rotating vortices called Ekman or Dean vortices. Fig. 7 illustrates the formation of a radial pressure gradient: it shows the pressure coefficient in the middle plane $\left(z / D_{\mathrm{h}}=0.5\right)$ of the concave-convex wall and of the convex-concave wall

$c_{p}=\frac{\left\langle p(\vec{x})-p_{0}\right\rangle}{\frac{1}{2} U_{\mathrm{b}}^{2} \rho_{\mathrm{b}}}$

where $p$ is the pressure at the corresponding wall and $p_{0}$ designates the pressure at the duct inlet. We first notice

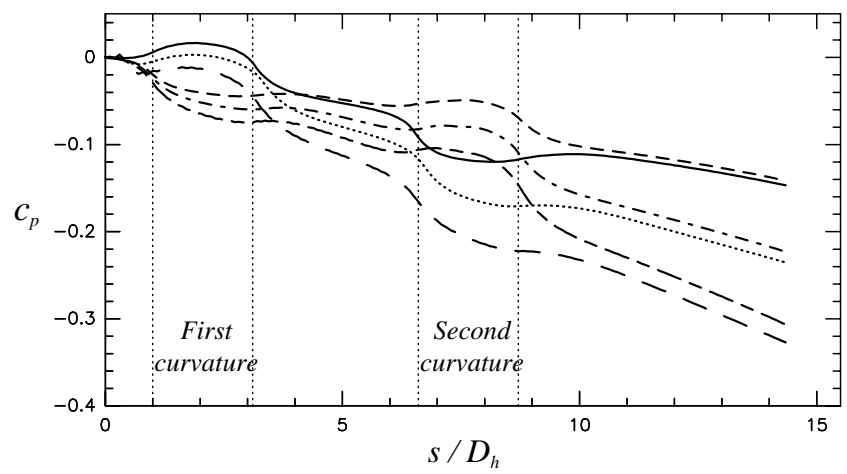

Fig. 7. Pressure coefficient $c_{p}$ at a fixed distance $z / D_{\mathrm{h}}=0.5$ from the lateral walls. Non-heated S-shape duct: $(-) n / D_{\mathrm{h}}=0$ (concave/ convex wall); (---) $n / D_{\mathrm{h}}=1$ (convex/concave wall). Heated $\mathrm{S}$ shape duct $\left(T_{\mathrm{h}} / T_{\mathrm{w}}=2\right):(\cdots) n / D_{\mathrm{h}}=0 ;(--) n / D_{\mathrm{h}}=1$. Heated Sshape duct $\left(T_{\mathrm{h}} / T_{\mathrm{w}}=3\right):(-\longrightarrow) n / D_{\mathrm{h}}=0 ;(--) n / D_{\mathrm{h}}=1$. The vertical dotted lines represent the beginning and end of the curved sections.

the global decrease of $c_{p}$ over the total length of the duct associated with the longitudinal mean pressure gradient within the duct. In the curved part of the duct situated near the inlet $\left(1<s / D_{\mathrm{h}}<3\right)$, the pressure coefficient is enhanced on the concave wall $\left(n / D_{\mathrm{h}}=0\right)$ owing to centrifugal effects (Fig. 7). Conversely, $c_{p}$ is reduced on the convex wall $\left(n / D_{\mathrm{h}}=1\right)$ : this indicates the presence of a radial pressure gradient directed from the convex wall towards the concave wall which drives a secondary flow from the concave wall to the convex wall. A similar pressure gradient is observed in the duct second curvature, but the concave wall now corresponds to $n / D_{\mathrm{h}}=1$ while the convex wall corresponds to $n / D_{\mathrm{h}}=0$. The progressive formation of the secondary mean flow is illustrated in Fig. 8 which shows the mean velocity vector projected on several cross-sections located at increasing values of $s$ along the duct. In the straight part of the duct near the inlet, the flow exhibits two weak counter-rotating vortices in the four corners of the duct. Within the first curved part, the radial pressure gradient induces a strong current concentrated near the side wall of the duct and directed from the concave towards the convex wall. Conversely, near the duct middle plane, the side wall current is compensated by a descending current away from the convex wall. Two large scale counterrotating vortices eventually form near the convex wall which are clearly visible in the straight part located between the two curvatures (location 3). In the second curved part, the side wall current is inverted and now flows from the top to the bottom in Fig. 8. By the same mechanism as the one previously described this yields a new pair of vortices in the vicinity of the convex wall (bottom of the figure, locations 4 and 5). In the final straight part of the duct, the two vortices near the top wall are still distinguishable but an extra pair of vortices 


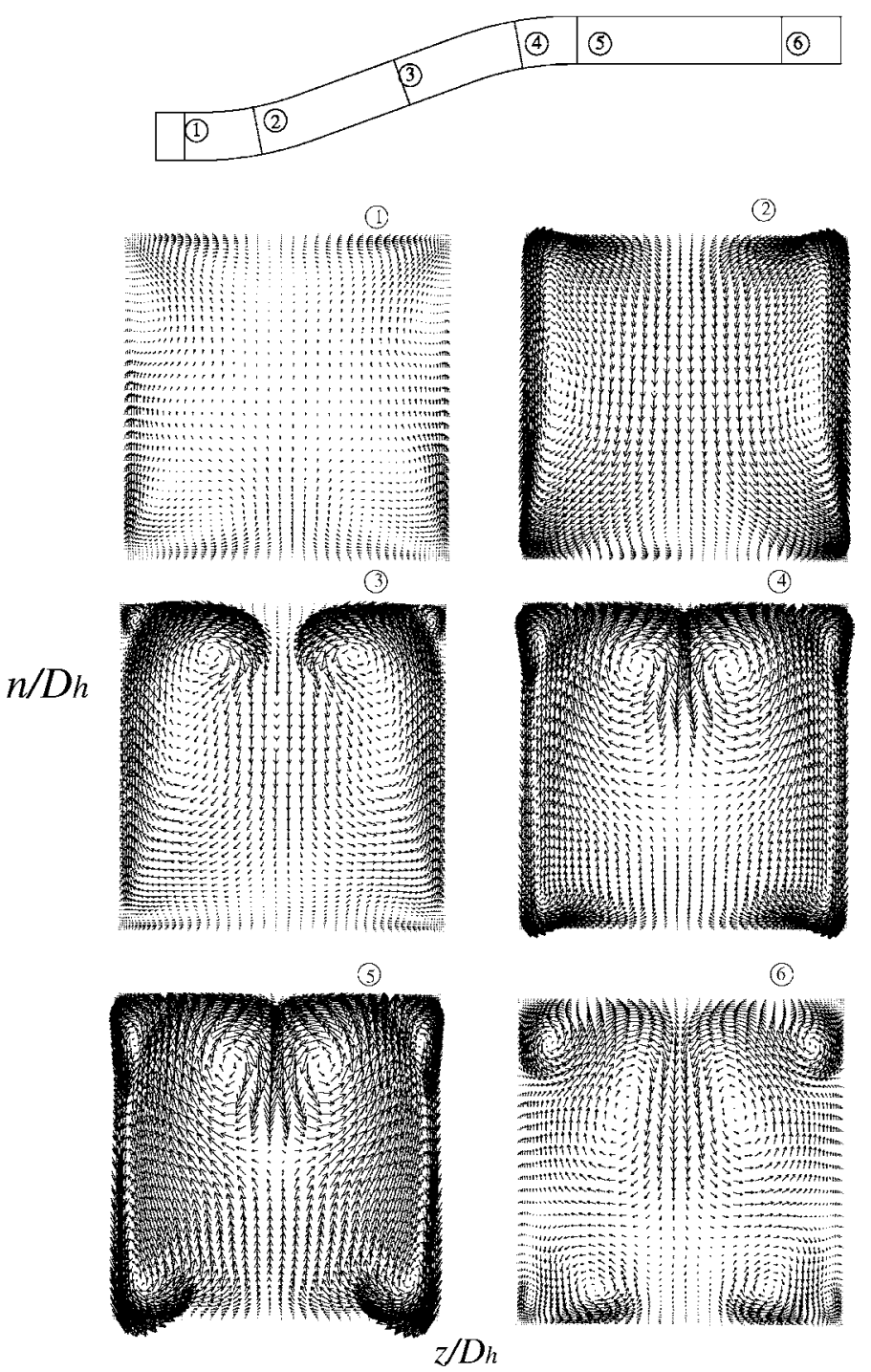

Fig. 8. Non-heated S-shape duct: visualization of the mean secondary flows for six downstream locations. The bottom wall corresponds to the concave-convex wall when the top wall corresponds to the convex-concave wall.

forms in the corners of this wall. The bottom wall also exhibits a system of four vortices. Fig. 9 displays a threedimensional view of the mean vortices forming within the duct that we will call Dean vortices. Note that the maximum intensity of the secondary flow is $22 \%$ of the bulk velocity. It is important to note that the instantaneous field is quite distinct from the mean field. Fig. 10 displays the instantaneous isosurfaces of positive $Q$ and shows that the large mean Dean vortices are indeed constituted of several fragmented elongated vortices which are unsteady. The presence of the radial pressure gradient strongly modifies the mean flow and therefore the turbulent production. We next consider the skin friction coefficient in the middle plane $\left(z / D_{\mathrm{h}}=0.5\right)$ of the concave-convex wall and of the convex-concave wall (see Fig.11): $c_{\mathrm{f}}=\frac{\left\langle\tau_{\mathrm{w}}(\vec{x})\right\rangle}{\frac{1}{2} U_{\mathrm{b}}^{2} \rho_{\mathrm{b}}}$

where $\tau_{\mathrm{w}}$ is the shear stress at the wall $\tau_{\mathrm{w}}=\mu\left(\frac{\partial\langle U\rangle}{\partial n}\right)_{n=0} . c_{\mathrm{f}}$ is non-dimensionalized by the averaged friction coefficient calculated on the centerline of the wall $c_{\mathrm{fm}}=\frac{1}{D_{\mathrm{h}} L_{\mathrm{w}}} \int_{0}^{L_{\mathrm{c}}} c_{\mathrm{f}}(s) \mathrm{d} s, L_{\mathrm{c}}$ is the length of the $\mathrm{S}$-shape duct. The downstream evolution of $c_{\mathrm{f}}$ exhibits the same characteristics than the coefficient experimentally measured by Bandyopadyhay and Ahmed (1993) (see their Fig. 5). Let us first consider the behaviour of $c_{\mathrm{f}}$ near the concave-convex wall. As pointed out by Bandyopadyhay and Ahmed (1993), the initial drop of $c_{\mathrm{f}}$ near the inlet is attributable to the radial pressure effect which tends to push the fluid away from the concave wall. Further downstream, the centrifugal effects push the 

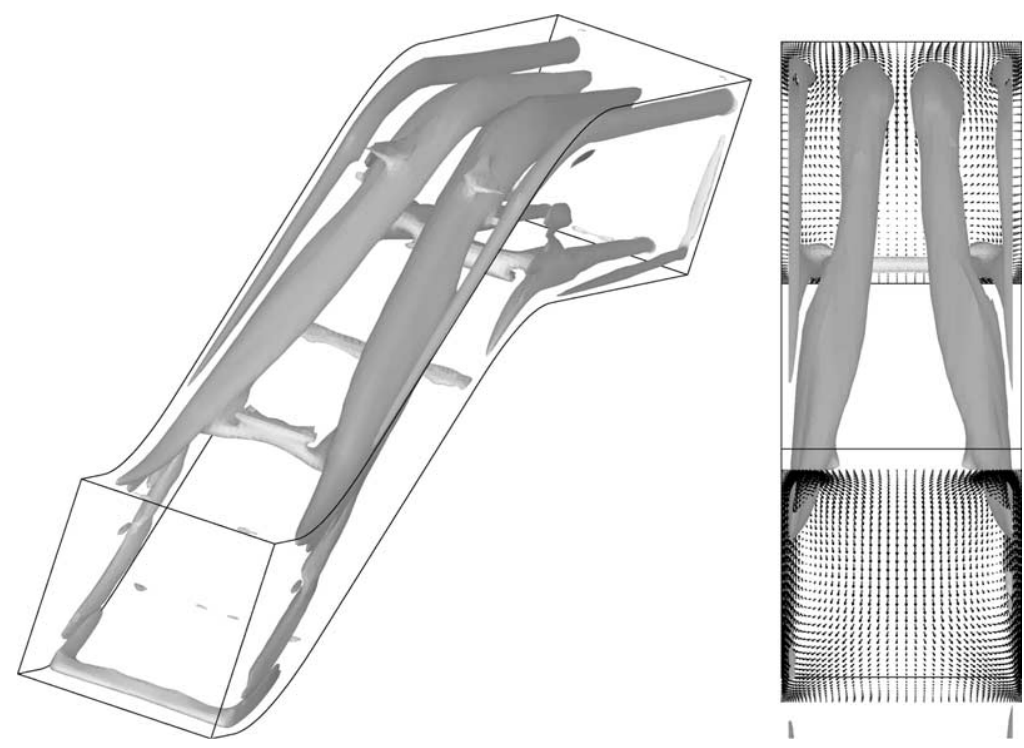

Fig. 9. Visualization of the Dean vortices through isosurfaces of the mean longitudinal vorticity in the S-shape duct.

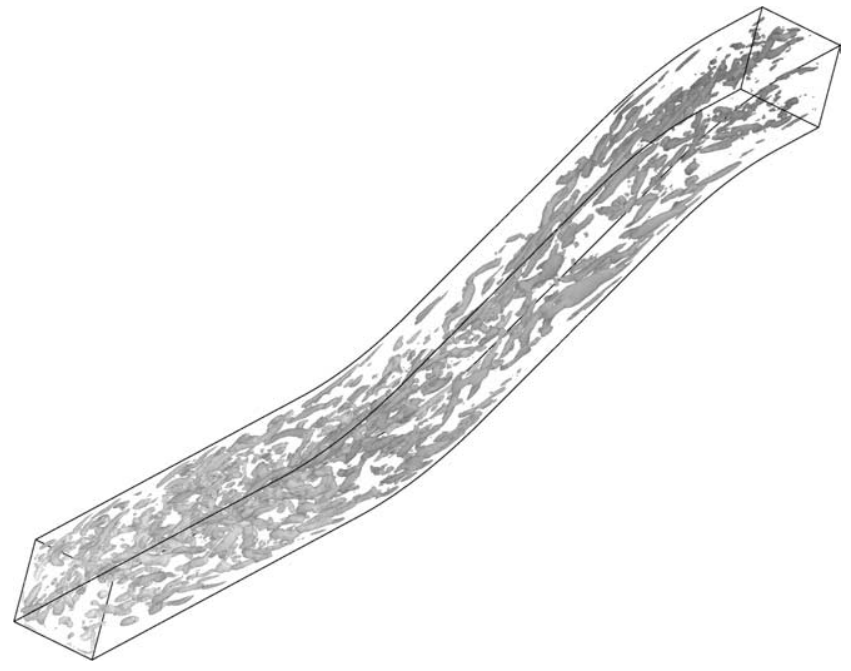

Fig. 10. Instantaneous visualization of the turbulent structures through positive $Q$ isosurfaces: $Q=0.7\left(U_{\mathrm{b}} / D_{\mathrm{h}}\right)^{2}$.

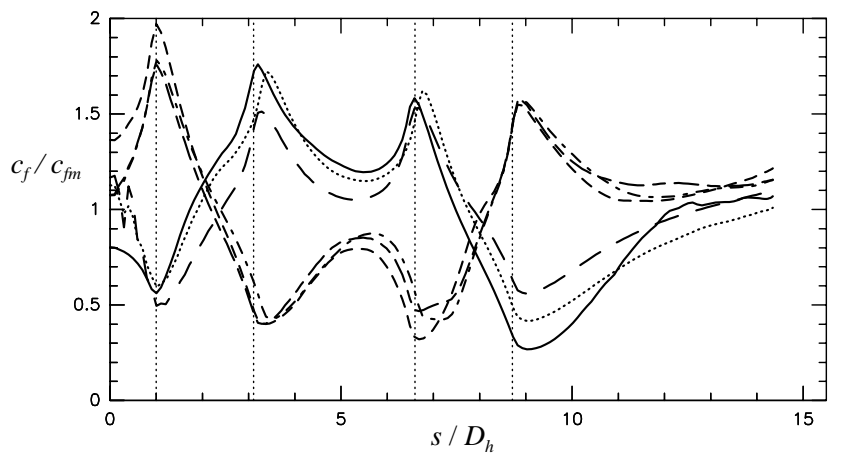

Fig. 11. Normalized friction coefficient: see legend on Fig. 7. flow towards the concave wall inducing an increase of $c_{\mathrm{f}}$. At the end of the bend, $c_{\mathrm{f}}$ tends to decrease towards the value it would have in a straight duct. Just before the second bend is reached, the pressure gradient is now directed towards the wall which now presents a convex curvature: this implies an increase of $c_{\mathrm{f}}$. Within the bend the convex curvature induces a strong decrease of the skin friction. The subsequent straight part is associated with an increase corresponding to the return for $c_{\mathrm{f}}$ to its straight duct value. Note that an overshoot is observed with a value of $c_{\mathrm{f}}$ larger than one: this is certainly attributable to the persistence of the Dean vortices after the curved region. Fig. 12 displays the profile of the turbulent kinetic energy per mass unit $k=\frac{1}{2}\left(\left\langle u^{2}\right\rangle+\right.$ $\left.\left\langle v^{\prime 2}\right\rangle+\left\langle w^{\prime 2}\right\rangle\right)$ at the beginning and at the end of each curved region of the duct. At the end of the first bend, we observe a high level of turbulence production owing to the velocity gradient enhancement near the concave wall. Conversely, the turbulence intensity clearly diminishes near the opposite convex wall due to the reduced production in that flow region. In the second bend, a turbulent kinetic energy peak appears near the concavely curved wall $\left(n / D_{\mathrm{h}} \approx 1\right)$. Note that the turbulence peak in the second curved region is driven away from the convex-concave wall $\left(n / D_{\mathrm{h}}=1\right)$ towards the duct core by the strong ejection induced by the Dean vortices present near this wall.

\subsection{Heated S-shape duct}

To investigate the mutual effect of the heating and of the Dean vortices, we have performed a LES of the same S-shape duct as previously described but the concaveconvex wall $\left(n / D_{\mathrm{h}}=0\right)$ is now heated by imposing a 

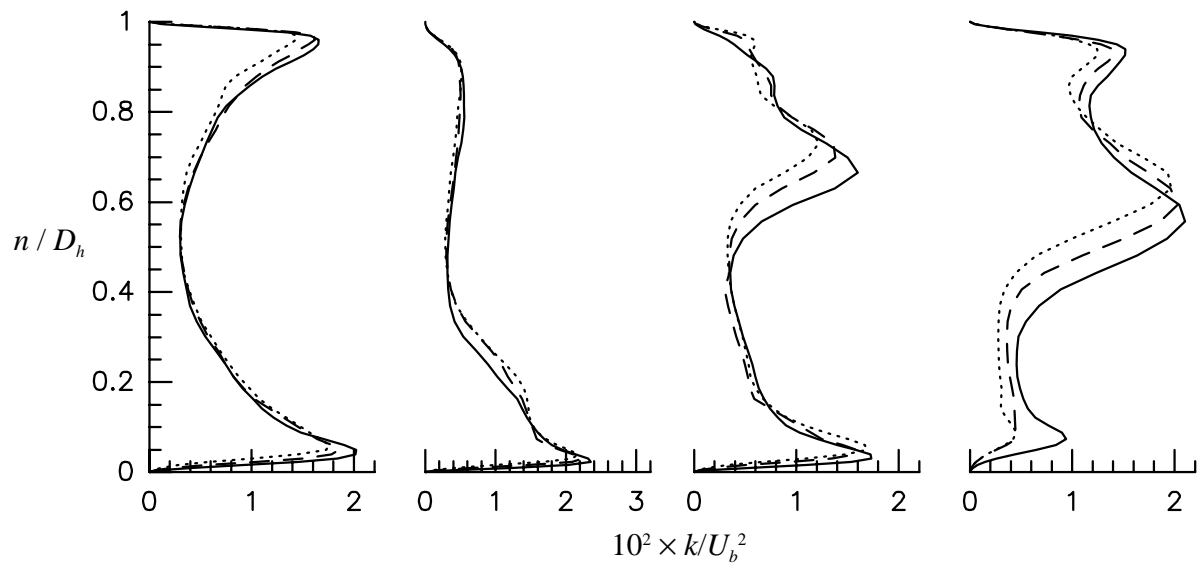

Fig. 12. Profiles of the turbulent kinetic energy at a fixed distance $z / D_{\mathrm{h}}=0.5$ from the lateral wall for four distances from the inlet, $s / D_{\mathrm{h}}=1,3.1,6.5$ and 8.7 (from the left to the right). Non-heated S-shape duct (-); heated S-shape duct $\left(T_{\mathrm{h}} / T_{\mathrm{w}}=2:(--)\right.$ and $\left.T_{\mathrm{h}} / T_{\mathrm{w}}=3:(\cdots)\right)$.

temperature $T_{\mathrm{h}}$ higher than the one of the other three walls $T_{\mathrm{w}}$. Two heating intensities are here considered corresponding with $T_{\mathrm{h}} / T_{\mathrm{w}}=2$ and $T_{\mathrm{h}} / T_{\mathrm{w}}=3$. As shown by $\mathrm{SM}$ and as we have seen in the first section devoted to the straight heated duct, the heating reinforces the ejection mechanism from the heated wall. Fig. 13 clearly shows that the same mechanism is here at play. By the end of the duct, the two Dean vortices observed near the heated wall (bottom wall on the figure) are enhanced both in size and intensity: their intensity is indeed increased by $1.5 \%$ for $T_{\mathrm{h}} / T_{\mathrm{w}}=2$ and $3 \%$ for $T_{\mathrm{h}} / T_{\mathrm{w}}=3$ in the second curvature as compared to the non-heated case. The presence of these vortices of larger size implies that the pair of Dean vortices near the opposite wall are squeezed and therefore exhibit a smaller and smaller size as the heating is increased. This reduction in size can be seen in Fig. 12: at the end of the second curve $\left(s / D_{\mathrm{h}}=8.7\right)$, the kinetic energy peak associated with the Dean vortices near the convex wall is progressively shifted towards the convex wall as the heating is increased. Conversely, the turbulent intensity is globally reduced near the heated wall due to the viscosity increase with temperature. This trend is clearly noticeable on the pressure and friction coefficients (see Figs. 7 and 11). The pressure drop within the duct is clearly enhanced when heating is applied ought to the larger energy dissipation. The skin friction on both concave-convex and convexconcave walls are similarly reduced indicating that the velocity gradients are reduced by viscosity.

In Fig. 14, we plot the downstream evolution of the heat flux at the heated-wall for both heated cases $T_{\mathrm{h}} / T_{\mathrm{w}}=2$ and $T_{\mathrm{h}} / T_{\mathrm{w}}=3$.

The heat flux is defined by the Nusselt number:

$N u(s, z)=H_{\mathrm{w}}(s, z) /\left(\kappa\left(T_{\mathrm{w}}\right) T_{\mathrm{w}} / D_{\mathrm{h}}\right)$

with $H_{\mathrm{w}}(s, z)=\left.\langle\kappa(T)\rangle \frac{\partial\langle T(s, n, z)\rangle}{\partial n}\right|_{n / D_{\mathrm{h}}=0}$ the mean wall heat flux. $\kappa(T)$ is the thermal conductivity obtained assuming the molecular Prandtl number is constant and equal to
0.7. The temperature dependence of the molecular viscosity is prescribed through a Sutherland empirical law (see e.g. White, 1991).

In the straight part of the duct near the inlet, strong ejections in the middle plane of the heated wall induce a strong reduction of the heat flux in that plane: indeed, the transport of the hot fluid away from the heated wall gives rise to a region where the temperature is quasihomogeneous (see SM). It is associated with weak temperature gradients and therefore weak heat fluxes. As we move downstream, the early decrease of the heat flux in the straight part is followed by a strong increase on the concave heated wall. This is attributable to the formation of the Dean vortices on the opposite convex wall which induce an intense transport of cold fluid away from the convex wall towards the concave hot wall: this amplifies the temperature gradients near the concave wall and consequently the heat flux. A diminution due to the oblique straight part follows, since the intensity of the secondary flow, driving cold fluid from the core toward the heated wall, decreases. When the heated wall becomes convex, the formation of two new counter-rotating structures near this wall enhance the ejection of hot fluid implying a sharp decrease of the heat flux. The heat flux increases in the final straight part of the duct due to the gradual attenuation of the Dean vortices near the heated wall. Note that the heat flux is maximal at the end of the first curve and minimal at the end of the second curve: the ratio between these two extrema is of the order of five. Fig. 15 shows the instantaneous heat flux on the hot wall. The strong values of the heat flux (dark grey) on the concave wall of the first curvature are seen to be concentrated near the wall's middle part and near the corners: these are due to cold fluid impacting the hot wall. In the straight part near the duct outlet, the appearance of the Dean vortices close to the hot wall leads to an enhancement of the heat flux close to the duct corners. As we move away from 

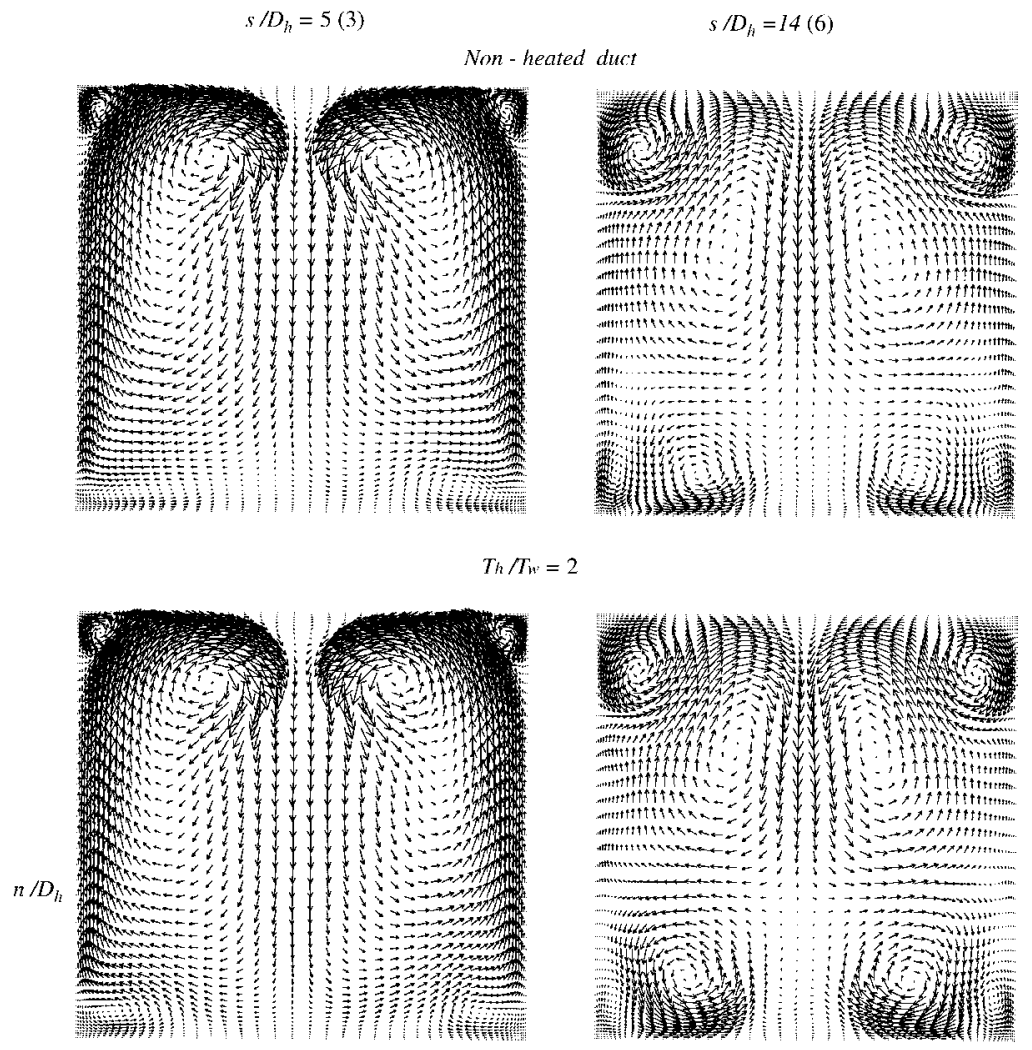

$T_{h} / T_{w}=3$
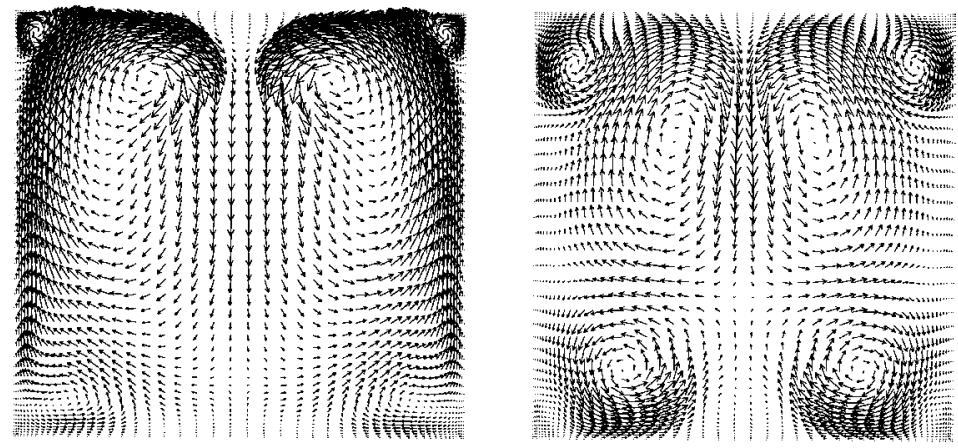

$z / D h$

Fig. 13. Comparison between the non-heated and the heated S-shape ducts: visualization of the mean secondary flows at locations three and six of Fig. 8.

the curved part, the attenuation of the Dean vortices leads to a progressive diminution of the heat flux intensity.

\section{Conclusion}

Through large-eddy simulation (LES) techniques, we have simulated the heat exchanges taking place within closed turbulent ducts of square cross-section. The case of a gas was considered here and we simulated the fully compressible Navier-Stokes equations.
We first considered the spatial development of the thermal boundary layer in a straight duct with one of its walls suddenly heated at a higher temperature than the other three walls. This study was motivated by the numerous applications where the flow in cooling passages undergoes a spatial growth before eventually reaching a fully developed turbulent state. As far as the heated straight duct is concerned, previous numerical studies by Salinas-Vasquez and Métais (2002) related to the fully developed turbulent regime have clearly demonstrated the dominant role played by the viscosity increase near the heated wall. This increase yields a 


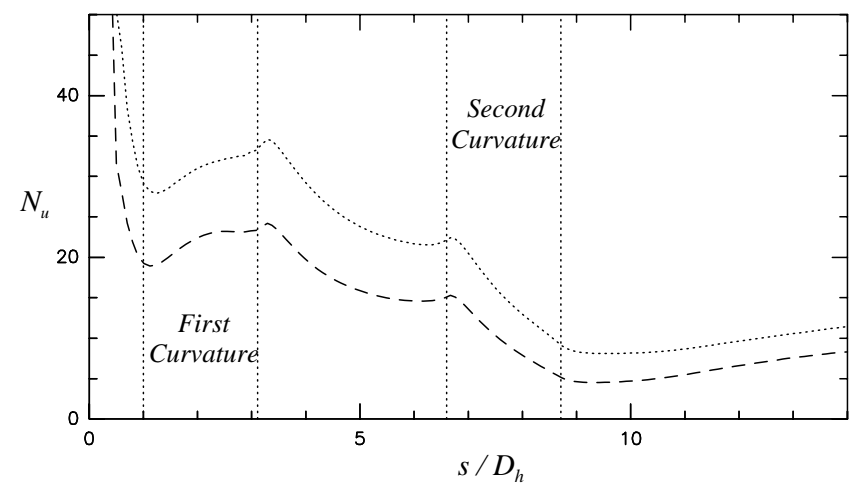

Fig. 14. Local Nusselt number at the heated wall at $z / D_{\mathrm{h}}=0.5$ and $n / D_{\mathrm{h}}=0$ in function of the streamwise direction $s / D_{\mathrm{h}}\left(T_{\mathrm{h}} / T_{\mathrm{w}}=2\right.$ $(--)$ and $\left.T_{\mathrm{h}} / T_{\mathrm{w}}=3(\cdots)\right)$.

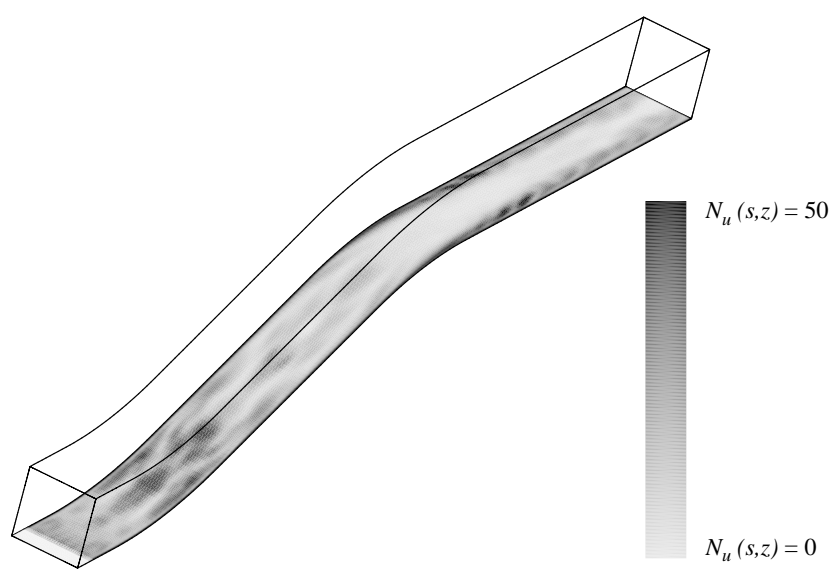

Fig. 15. Local Nusselt number at the heated wall $\left(T_{\mathrm{h}} / T_{\mathrm{w}}=3\right)$.

global augmentation of the size of the turbulent structures which leads to the widening of the low- and highspeed streaks system and to the formation of a single ejection of fluid away from the heated wall localized near the central plane of the latter. This ejection is associated with an enhancement of the transverse mean secondary flow characteristic of the flow in a closed duct of square cross-section. The advantage of simulating the spatial growth of the flow relies upon its ability to investigate the progressive downstream formation of the flow turbulent structures.

Although the simulation displays the progressive enhancement of the size of the characteristic turbulent structures of the boundary layer and a progressive amplification of the transverse secondary flow, the present LES showed that the establishing of a fully developed thermal state requires a downstream distance longer than $31 D_{\mathrm{h}}$ which was considered here.

In several industrial configurations of heat exchangers, heating and curvature effects are often simultaneously present. To study these combined effects, we next simulated a S-shape duct which presents the interesting particularity of exhibiting two successive inverted curvatures. Indeed, one of the curved wall presents a concave-convex curvature while the opposite curved wall has a convex-concave curvature. In agreement with the previous experimental works, we found that the pressure gradient between the inner and outer wall of the curved section leads to the apparition of intense counter-rotating Dean vortices associated with an intense transverse flow with a maximum intensity of $22 \%$ of the bulk velocity. These are found to be located near the convex wall. Furthermore, we investigated how these Dean vortices are affected by the second curvature. In the heated case, we varied the heating intensity to determine the mutual interaction between the heating and the Dean vortices. The heat flux on the heated wall is found to be strongly influenced by the various vortices: impinging regions with the heated wall indeed correspond with important heat fluxes, when ejections are associated with low heat fluxes. Furthermore, the heating is seen to enhance both the size and intensity of the Dean vortices situated close to the heated wall. Some previous experimental works have been devoted to Sshape duct in the non-heated case but, to our knowledge, no previous work has been devoted to the heated case. The present work may hopefully be used as a test case for turbulence modelling approaches which heavily rely on empirical laws.

\section{Acknowledgements}

All the numerical computations were carried out at the "Institut du Développement et des Ressources en Informatique Scientifique".

\section{References}

Bandyopadyhay, P.R., Ahmed, A., 1993. Turbulent boundary layers subjected to multiple curvatures and pressure gradients. J. Fluid Mech. 246, 503-527.

Bruns, J.M., Fernholz, H.H., Monkewitz, P.A., 1999. An experimental investigation of a three-dimensional turbulent boundary layer in an 'S'-shaped duct. J. Fluid Mech. 393, 175-213.

Dubief, Y., Delcayre, F., 2000. On coherent-vortex identification in turbulence. J. Turbulence 1, 001 .

Gavrilakis, S., 1992. Numerical simulation of low Reynolds number turbulent flow through a straight square duct. J. Fluid Mech. 244, $101-129$.

Gottlieb, D., Turkel, E., 1976. Dissipative two four methods for timedependent problems. Math. Comput. 30, 703-723.

Grinstein, F.F., 1994. Open boundary conditions in the simulation of subsonic turbulent shear flows. J. Comput. Phys. 115, 4355.

Hunt, J., Wray, A., Moin, P., 1988. Eddies, stream, and convergence zones in turbulent flows. Center for Turbulence Research Rep., Technical Report CTR-S88. 
Kennedy, C.A., Carpenter, M.H., 1997. Comparison of several numerical Methods for simulations of compressible shear layers. NASA, Paper 3384.

Lesieur, M., Métais, O., 1996. New trends in large-eddy simulation of turbulence. Ann. Rev. Fluid Mech. 28, 45-82.

Mees, P.A.J., Nandakumar, K., Masliyah, J.H., 1996. Secondary instability of flow in a curved duct of square cross-section. J. Fluid Mech. 323, 387-409.

Poinsot, T., Lele, S., 1992. Boundary conditions for direct simulations of compressible viscous flows. J. Comp. Phys. 101, 104-129.

Poinsot, T., Veynante, D., 2001. Theoretical and Numerical Combustion. Edwards.
Salinas-Vasquez, M., Métais, O., 2001. Large-eddy simulation of a spatially growing thermal boundary layer in a turbule nt square duct. Direct and Large-Eddy Simulation IV, Kluwer Academic Publishers, 277-284.

Salinas-Vasquez, M., Métais, O., 2002. Large-eddy simulation of the turbulent flow through a heated square duct. J. Fluid Mech. 453, 201-238.

Sotiropoulos, F., Ventikos, Y., 1998. Flow through a curved duct using nonlinear two-equation turbulence models. AIAA Journal 36 (7), 1256-1262.

White, F.M., 1991. Viscous Fluid Flow. McGraw-Hill. 